\title{
SHEM TOB IBN FALAQUERA E LA FILOLOGIA EBRAICA MEDIEVALE A PROPOSITO DI DUE LIBRI RECENTI *
}

MAURO ZONTA

Pavía

I. Premessa

Il contributo che segue mira a sottolineare il ruolo svolto dal filosofo sefardita Shem Tob ben Yosef ibn Falaquera (1225-post 1290) nella trasmissione e nella divulgazione della filosofia e della scienza araba. Questa sua attività si dispiega in due campi diversi ma complementari: l'ambito "religioso", ove l'autore si propone, con i suoi scritti, di favorire l'accettazione della filosofia da parte dei suoi correligionari -in un secolo che, come è noto, vide il Giudaismo, e quello spagnolo e provenzale in particolare, acceso dalla controversia su Maimonide; e l'ambito più strettamente «scientifico", nel quale il nostro si sforza di creare strumenti di conoscenza che uniscano i pregi della chiarezza a quelli di una vasta ed enciclopedica dottrina, che renda familiari al pubblico ebraico i testi aristotelici e i loro commentatori.

Il primo dei due campi è rappresentato tra l'altro, all'interno della vasta produzione letteraria di Falaquera, da un trattatello sulla disputa tra «scienza" e «fede». La recente edizione di questo trattatello offre il destro non solo per alcune riflessioni sul pensiero

* S. Harvey, Falaquera's Epistle of the Debate. An Introduction to Jewish Philosophy, "Harvard Judaic Texts and Studies" VIII. Harvard University Press. Cambridge (Mass.), London 1987, pp. XVI + 155. H. J. DrossaART Lulofs - E. L. J. POorTmaN (edd.). Nicolaus Damascenus, De plantis. Five Translations, "Verhandelingen der Koninklijke Nederlandse Akademie van Wetenschappen, Afdeeling Letterkunde", n. r. 139 ("Aristoteles Semitico-Latinus"), North-Holland Publishing Company, Amsterdam - Oxford - New York 1989, pp. XVI + 729. 
dell'autore in proposito - alla luce dei suoi rapporti con le fonti arabe, e con Averroè in particolare-, ma anche per riproporre la questione di una corretta e, soprattutto, solidamente fondata edizione dei testi ebraici medievali. La necessità di questa rigorosa base filologica emerge in tutta la sua evidenza nella disamina del secondo dei due libri qui presentati: la ricostruzione di un testo scientifico greco conservato solo grazie alle sue traduzioni orientali (siriaca, araba, ebraica), una delle quali è appunto inclusa nella più ampia opera enciclopedica del nostro autore. Appare chiaro, in questo caso, il ruolo di Falaquera come divulgatore scientifico, ma è anche chiara la sua fondamentale importanza nella filologia ebraica medievale: il suo contributo è infatti determinante nell'illuminare punti altrimenti oscuri della storia del testo, alla luce, tra l'altro, di nuove testimonianze sfuggite ai curatori dell'edizione.

\section{II. 'IGGERET HA-WIKKÛAH: FILOSOFIA E FILOLOGIA}

L'opera di Shem Tob ibn Falaquera è stata negli ultimi anni oggetto di due studi che analizzano alcuni aspetti della sua personalità di studioso, e rendono disponibili nel contempo testi sinora inediti o male editi. Si tratta di R. Jospe, Torah and Sophia, Cincinnati 1988 - un ampio ma disorganico saggio, sottotitolato The Life and Works of Shem Tov Ibn Falaquera, del quale abbiamo già discusso altrove (Henoch 12 [1990] 207-226)-, e del libro di S. Harvey qui presentato, che è dedicato all''Iggeret ha-Wikkuah [= IhW] (Il trattato del dibattito), un'operetta giovanile ' del nostro sul problema dell'accordo tra Legge e Sapienza straniera ההסכמה אשר בין) (חסיד) (חתור) e un «filosofo" (מח).

Falaquera è parso, ad entrambi gli studiosi, un tipico difensore della necessità dell'accordo tra religione giudaica e filosofia grecoaraba — che include naturalmente anche la scienza-, nel pieno delle controversie dei secc. XIII-XIV sulla compatibilità tra le opinioni sostenute da Maimonide nella Guida dei perplessi e le dottrine tradizionali giudaiche su problemi quali la creazione del mondo,

1 Per la datazione relativa dell'IhW all'interno della produzione di Falaquera, v. la cronologia proposta da R. JOSPE, op. cit., pp. 31-33. 
l'onniscienza di Dio, ecc. ${ }^{2}$. In particolare, lo studio di Harvey mira ad attualizzare questa problematica, inserendola nel quadro dell'armonizzazione tra "fede» e "scienza», e cercando di mostrare come il pensiero ebraico medievale possa dare una risposta alle domande del lettore moderno su questo punto.

Intento di Harvey è dunque, innanzitutto, quello di approfondire questo speciale aspetto del pensiero di Falaquera: perciò, all'edizione critica del testo dell'IhW (pp. 53-80), preceduta da una traduzione inglese dell'opera (pp. 11-52), fanno seguito tre studi, dedicati rispettivamente alle fonti dell'IhW (pp. 83-101), alla posizione di Falaquera e di altri pensatori dell'ebraismo medievale sul problema in questione (pp. 102-127), e ai motivi per cui il nostro cessò la sua giovanile attività di poeta (pp. 128-132).

Chiudono l'opera un glossario ebraico-latino-inglese dei termini filosofici occorrenti nell'IhW (pp. 133-136), una bibliografía (pp. 137-147), e un utile e ben organizzato indice non solo dei passi biblici e rabbinici, ma anche di quelli degli autori greci, arabi e giudaici citati nel corso del libro (pp. 148-155).

\section{Un protrettico filosofico}

L'introduzione (pp. ix-xiv) mira a puntualizzare due importanti questioni preliminari.

Innanzitutto, vengono chiariti i motivi che spinsero Falaquera a redigere l'IhW: gli intenti di divulgazione filosofica da lui perseguiti dovevano spingerlo a giustificare, in via preliminare, l'utilità, anzi, la necessità dello studio della filosofia per lo studio della Torah. In questo senso, il trattatello è un'intoduzione alla filosofia, o, per meglio dire, un protrettico, che il nostro conclude preannunciando la pubblicazione di scritti più impegnativi, nei quali si tratteranno «i segreti delle scienze» e «i pensieri dei filosofi» סודות החכמות) סברות הפילוסופים : p. 79,1-2).

In secondo luogo, Harvey opportunamente difende il nostro da quelle accuse di scarsa originalità che molti storici moderni hanno avanzato contro di lui, pur riconoscendogli una notevole competenza

${ }^{2}$ Cfr. il quadro di tali controversie delineato da R. JOSPE in un saggio non citato da Harvey, "Faith and Reason: The Controversy over Philosophy", in R. JOSPE - S. WAGNER (edd.), Great Schisms in Jewish History, New York 1981, 73-117 (per Falaquera, v. specialmente 103-105). 
in tutte le discipline da lui affrontate. Non solo è dubbio che l'originalità sia un criterio valido per giudicare un filosofo, ma è certo che Falaquera, proprio limitandosi a tradurre, compendiare e insegnare i testi dei suoi predecessori, ci offre un quadro documentatissimo dei grandi problemi filosofici del suo tempo, con la maestria di un vero storico, che nulla ha da invidiare a molti suoi colleghi moderni; anzi, li supera per la diretta e più vasta conoscenza di parecchie delle sue fonti. Bene conclude Harvey: "I know of no person alive today from whom the serious student can learn more about the problems and concerns of medieval philosophy" (p. xiv).

Purtroppo, queste efficaci note di premessa non trovano sempre un adeguato sviluppo nei tre studi successivi. Il terzo di essi è del tutto inconsistente, e non apporta alcuna novità al tema ormai trito del "ruolo della poesia in Falaquera" (per cui v. R. Jospe, Torah and Sophia, 11-17, nonchè Henoch 12 [1990] 213-214); ma anche gli altri due soffrono di una sostanziale limitatezza di prospettive, che tradisce il carattere talora "apologetico" del libro di Harvey.

\section{Il rapporto con le fonti arabe}

Lo studio sulle fonti è dedicato al rapporto tra l'IhW e il Fasl almaqāl (Trattato decisivo [sul legame tra religione e sapienza]), che già da tempo era stato identificato come fonte principale dell'operetta. Il rapporto tra i due testi era stato accuratamente esaminato da $G$. Dahan nello studio preliminare alla sua edizione della versione latina dell'IhW (Sefarad 39 [1979] 47-85; 237-264); ora Harvey, con argomentazioni non dissimili ${ }^{3}$, mostra che il Fasl è piuttosto un modello di ispirazione, cui Falaquera attinge soprattutto certi schemi argomentativi, senza mai giungere a una vera «traduzione».

La differenza fondamentale tra le due opere risiederebbe, secondo Harvey, nello spiccato carattere "giudaico" dello scritto di Falaquera, ottenuto mediante l'introduzione di elementi propri della tradizione religiosa, che nulla hanno a che vedere con l'impostazione filosoficolegalistica del trattato averroiano. A tale proposito, Harvey dedica un'apposita appendice al pensiero di L. Strauss, che individuò in un'altra opera falaqueriana, la Resit Hộmâ, la presenza di analoghe

\footnotetext{
${ }^{3}$ Non sembra peraltro molto corretto che S. HARVEY dichiari: "Dahan's work supports my conclusions on the influence of Averroes' Decisive Treatise" (p. 137), giacché l'opera di Dahan è stata pubblicata ben otto anni prima della sua.
} 
rielaborazioni "giudaizzanti» delle fonti arabe (MGWJ 80 [1936] 96106).

In realtà, come ha ben rilevato $\mathrm{M}$. Mahdi (nella sua introduzione all'edizione della Falsafat Ariștūtālis di al-Fārābīi, Beirut 1961, p. 37), la vera "differenza tra Averroè e Falaquera sta nel fatto che Averroè si sforza di affermare il legame tra filosofia e legge islamica, mentre Falaquera si sforza di affermare il legame tra filosofia e legge giudaica"; è stato anche notato che il nostro si sforza di dare alla propria opera una patina "giuridica" che la avvicini al Fasl, ricorrendo, p. e., a un riferimento alla Mišnâ nella discussione sul concetto di כפירה, "miscredenza" (v. pp. 59,15 ss.; cfr. G. Dahan, op. cit., pp. 72-73). Come ancora Dahan ha osservato, sono i puntuali riferimenti alla Guida di Maimonide che danno all'IhW il suo carattere "giudaico" (ibid., pp. 69 ss.). D'altra parte, Falaquera dimostra anche nell'IhW la propria ampiezza di orizzonti: il racconto della disputa tra Abramo e Nemrod (p. 68,14 ss.) è ripreso proprio dal testo del Corano (II, 260) ${ }^{4}$.

Il secondo studio di Harvey ("Falaquera on the Importance of Philosophy for Understanding Torah") affronta più in generale il problema del ruolo affidato dal pensiero ebraico medievale alla filosofia. Nell'IhW come nel Sefer ha-Mĕbaqqeš, opere di carattere "propedeutico" e "popolare", Falaquera fa mostra di aderire ai precetti talmudici che vietano la rivelazione dei "segreti» della fisica e della metafisica (cfr. bHagîgâ 11b); Harvey ne deduce che Falaquera assegna alla filosofia il mero ruolo di ancilla Legis: "Falaquera's intention is not to show that philosophy leads to the ultimate truths, but rather to show that the Torah read with the help of philosophy leads to these truths" (p. 119). Fine dichiarato dell'analisi di Harvey è qui l'attualizzazione del pensiero di Falaquera per trovare una risposta alle esigenze del Giudaismo moderno ( $\mathrm{v}$. l'excursus "So what?" alle pp. 122-127); tuttavia, se pure questo appiattimento su una posizione di assoluta fedeltà alla tradizione vale per l'IhW, non si può estenderlo anche alle opere della maturità (Dé $\hat{t} \underline{t}$ ha-Filosofim, Moreh ha-Moreh), dove Falaquera dedica ampio spazio ai più ardui problemi di fisica e metafisica, nel solco delle teorie di Averroè e al-Fārābì.

\footnotetext{
${ }^{4} \mathrm{La}$ fonte di questo passo è appena accennata da Harvey, con un rimando a $\mathrm{L}$. GinZBerg, The Legends of the Jews, Philadelphia 1909-1938, v, p. 218, n. 50: in realtà, l'unica fonte nota di questa tradizione "giudaica" è appunto il Corano, non trovandosene traccia in altri testi ebraici (G. DAHAN, op. cit., p. 66).
} 
3. Applicabilità dei principi ecdotici classici ai testi ebraici medievali: il caso dell''Iggeret

Di fatto, il maggior merito di Harvey è stato di aver finalmente approntato un'edizione affidabile dell'operetta di Falaquera. In effetti, come egli chiarisce nel capitolo "The Establishment of the Text" (pp. 3-10), mentre la prima pubblicazione a stampa, inserita dal bibliofilo Ișhaq ben Abraham 'Aqrish nella sua raccolta miscellanea 'Iggeret 'Ôgeret (Costantinopoli, s.d., ma verso il 1577), conteneva diversi errori ed era assai difficile da reperire, la seconda, curata da Gershom ben Beșalel Cohen a Praga nel 1610, risultava contaminata da considerevoli rimaneggiamenti stilistici, condotti dall'editore sul testo di Costantinopoli, al fine di attenuare l'asprezza della disputa. A questa seconda edizione si rifaceva a sua volta la terza, preparata da A. Jellinek (Vienna 1875), le cui mende erano state rilevate già da M. Steinschneider in Hebräische Bibliographie 15 (1875) 41-43: le notevoli differenze riscontrate tra quest'ultima edizione e i manoscritti furono peraltro spiegate da J. Dan (ספרד אוצר יהודי] 6 [1963 42-46) con il cumularsi dei suddetti interventi «ideologici» con gli infelici tentativi dei copisti, volti a restaurare un testo già corrotto.

Sulla base di queste considerazioni, Harvey ha proceduto a ripubblicare il testo. La sua edizione ha certamente restituito un testo molto più vicino all'originale di tutti quelli che l'hanno preceduto, rendendolo finalmente leggibile; spiace però notare che non sempre sia stata condotta con il necessario scrupolo filologico. Dei dodici testimoni noti, l'editore ne ha esaminati solo sette, senza peraltro aver prima proceduto alla recensio, sicché la sua scelta resta del tutto arbitraria. In effetti, anziché tracciare uno stemma codicum (abitudine che dovrebbe ormai estendersi - quando possibile- anche al di fuori della filologia classica: v. G. Contini, Breviario di ecdotica, Milano-Napoli 1986 [= Torino 1990], pp. 65-66), Harvey propone solo alcune confuse considerazioni sulle relazioni tra i diversi mss, senza fondarle su un metodico rilievo degli errori significativi, secondo le più elementari regole filologiche: tra l'altro, egli si è rifiutato di introdurre nel testo emendationes ope ingenii (p. 9: "I have not offered any readings, grammatical or otherwise, of my own"). Comunque, sulla base del confronto tra le sue osservazioni e le costellazioni delle varianti riportate in apparato, si può tracciare il seguente «albero" dei testimoni esaminati: 


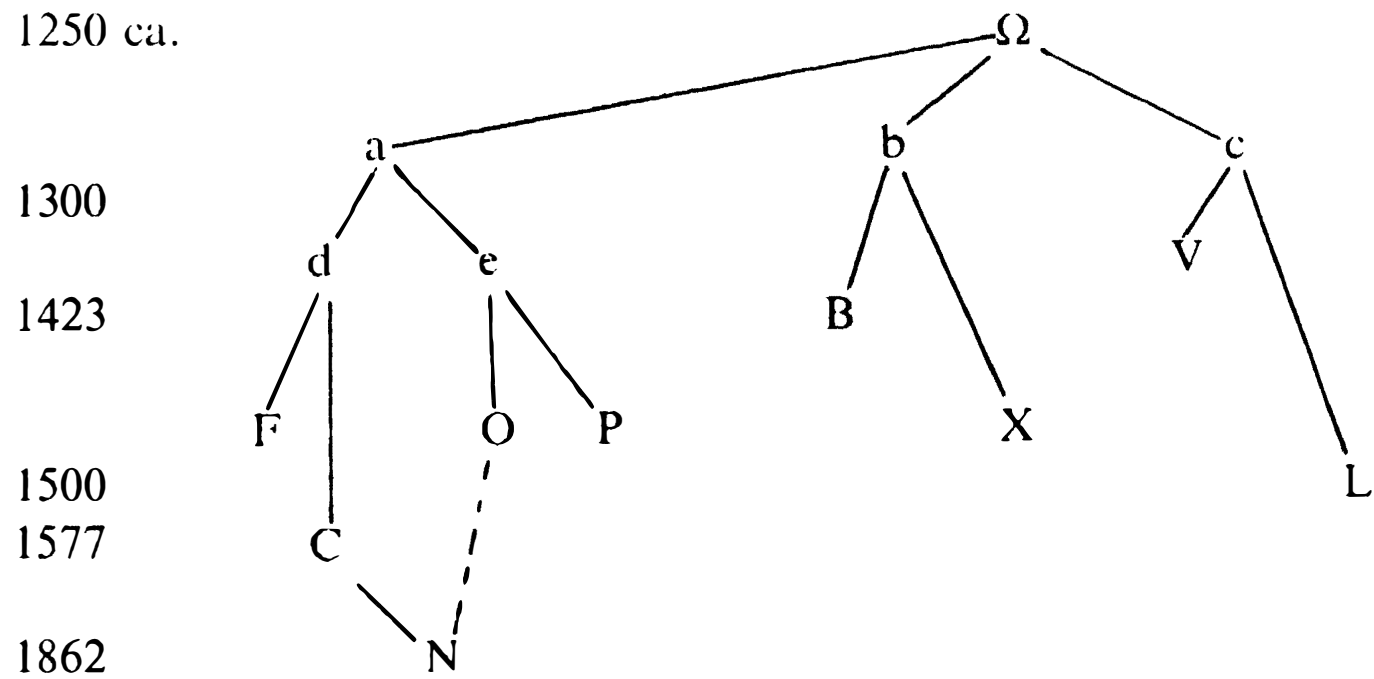

$\Omega=$ originale; $\mathrm{O}=$ Oxferd, Bodl., Mich 279; $\mathrm{X}=$ Oxford, Bodl., Mich. 460; $\mathrm{F}=$ Oxford, Bodl., Marsh 410 (framm.); B = Londra, B. M., Add. 26925; P = Parigi, B. N., hébr. 893; $\mathrm{V}=$ Roma, Vat. ebr. 339; $\mathrm{N}=$ New York, Jewish. Theol. Sem., $\mathrm{n}$. 2422; L = trad. lat. anonima, ms Parigi, B. N., lat. 6691 (= ed. Dahan); C = ed. Costantinopoli 1577.

Purtroppo, questo stemma può avere solo una validità provvisoria, restando ignota la collocazione degli altri cinque testimoni: tra di essi, è opportuno segnalare il ms di Monaco, Bay. Staatsbibl., hebr. 402, che, insieme a B e a V, abbraccia tutta la trilogia costituita dall'IhW, dalla Reš́t hịoknâ e dal Sefer ha-Ma'alôt, le due opere la cui prossima composizione era stata annunciata dal nostro in chiusura dell'"Epistola» (v. p. 79). Detto ms è infatti imparentato con $\mathrm{V}$ (ritenuto da Harvey stesso il più antico e più affidabile tra $\mathrm{i}$ mss da lui impiegati), come si può constatare da una collazione di quest'ultimo testimone con il testo edito della Resît di M. David, Berlin 1902), basato, tra l'altro, sul ms monacense s.

D'altra parte, l'esistenza del sub-archetipo a è dimostrata da almeno un errore congiuntivo e separativo di $\mathrm{P}, \mathrm{C}$ e $\mathrm{O}$ : si tratta della lezione משהביאך, sostituita a quella corretta יש מי הביאו a p. 61,23. La presenza di un archetipo comune, invece, non può essere confermata; comunque, se lo stemma proposto è valido, la recensio

${ }^{5}$ Il ms $\mathrm{V}$ e quello di Monaco condividono infatti diversi errori congiuntivi (cfr. p. e. l'aggiunta di ומסבריהם a p. 27,30, l'omissione di איבריהם a p. 22,13, l'aggiunta di אין צריך החכמה סכלית a p. 58,13). Tali errori sono assai numerosi, e facilmente identificabili grazie al confronto con le fonti della Resît Hoknmâ (l'Ihssăa al-‘ulūm di al-Fārābî e gli Aqsām al'ulüm al-'aqliyyah di Avicenna). 
dell'IhW presenterebbe il raro vantaggio di consentire la scelta delle varianti sulla base di un criterio puramente meccanico, e, dunque, prettamente lachmanniano.

Questa sostanziale carenza di basi filologiche non toglie che l'edizione e la traduzione di Harvey risultino soddisfacenti almeno come strumento di lavoro. Solo pochi rilievi si possono avanzare:

- p. 56,11 del testo = p. 16,7-8 della traduzione: il passo והשוי non va interpretato "the second was a scholar, who at times engaged in the study of the Law and at times in the study of science", bensi più precisamente, come fa il traduttore latino, "alius autem erat vir Philosophus, semel Legi et bis Philosophiae incumbens" in riferimento alla differenziazione tra i tempi dello studio della Torah e gli studi profani di cui fa menzione Maimonide, in Sefer ha-maddac, III, 1, 12 (v. G. Dahan, op. cit., p. 240 n. 11);

- p. 62,8 del testo $=$ p. 25,6 e n. 29 della trad.: la lezione ההצעות («fondamenti») respinta dall'editore in apparato è certamente da preferirsi a quella accolta, seppur dubbiosamente, nel testo, ההשגות («percezioni, nozioni»). Quest'ultima, infatti, benchè sia accolta dal ms più antico (V), è il risultato di una palese trivializzazione, mentre la prima non solo è sostenuta da due famiglie di mss su tre (quelle facenti capo ai sub-archetipi $a$ e $b$ ), ma è anche confermata dalla analoga lezione di p. 73,13;

- p. 66,13-14 del testo = p. 30,15-16 della trad.: il testo ebraico sembra prima facie corrotto, specialmente per un brusco cambiamento di soggetto. Tuttavia, esso è sicuramente corretto, come dimostra il confronto con un passo pressoché identico dell' $I h s \bar{a}^{\prime}$ al'ulūm di al-Fārābì (v. ed. Amin, Cairo 1968, p. 67,10-11), e la diversa traduzione che di questo stesso passo Falaquera dà in Rešit Hiokmâ p. 31,19-20:

Rešît Hoknmâ

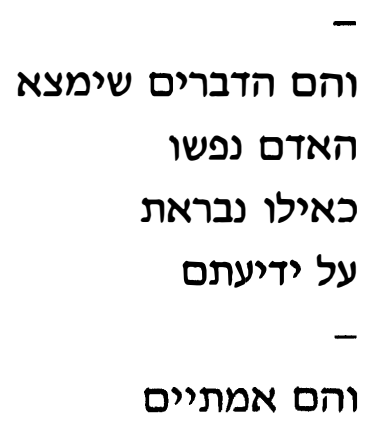

'Ihṣāa' al-‘ulūm

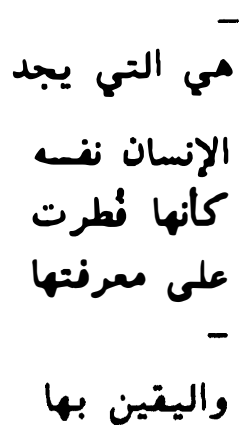

'Iggeret ha-Wikkûạ

כי אלו המושכלות

הם אשר נמצא

נפשותינו

כאלו נוצרו

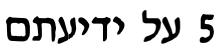

מתחלה ויודעות

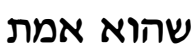


(alla 1. 3, la lezione dell'ed. David, בנפשו, è stata corretta in נפשו, com'è nei mss di Monaco, Bay. Staatsb., hebr. 42 e 405, nonchè nei mss V [f. 36r,6] e B [f. 13v,1], non impiegati nell'edizione).

L'interpretazione corretta di questo passo è dunque: "Quei concetti sono quelli per la cui conoscenza sembra che noi siamo stati creati fin dal principio, sapendo che sono veri».

Nel complesso, il libro di Harvey, un'operetta senza pretese ma ben organizzata, è una valida guida alla lettura e alla comprensione di uno scritto anch'esso "leggero" e di carattere divulgativo come l'Iggeret ha-Wikkûah, purché ci si ricordi che solo di una «introduzione" si tratta, e che inoltrarsi nello studio delle successive opere di Falanquera richiede certamente un maggiore sforzo di approfondimento storico e filologico. È questo, appunto, il caso delle $D e^{c} \hat{o} \underline{t} h a-$ Filosofim, certo il più ampio scritto del nostro, che, come una grande miniera di scienza greca e araba, va dischiudendo progressivamente agli studiosi testimonianze di grande interesse storico, talora uniche ${ }^{6}$. Vedremo ora una nuova prova -e senz'altro non l'ultimadell'importanza di questo testo.

\section{Il DE PLANTIS DI Nicola DI Damasco E la sua tRadizione ebraica}

Le traduzioni medievali di Aristotele, che portarono a conoscenza dell'Oriente e dell'Occidente non grecofono i testi della più alta auctoritas della scienza del tempo, risvegliano sempre più l'attenzione degli studiosi. La collana "Aristoteles Semitico-Latinus", iniziatasi nel 1971 con l'edizione della versione araba del De generatione animalium ', e attualmente diretta da H. J. Drossaart Lulofs e H. Daiber, testimonia la crescita di questo interesse: a suscitarlo non è tanto la speranza (sinora rivelatasi vana) di recuperare scritti altrimenti perduti dello Stagirita, ma piuttosto quella di individuare gli annelli della "catena di trasmissione" della scienza greca nel Medioevo - una trasmissione le cui vicende si identificano per lo più con la storia e la fortuna dei testi aristotelici.

Questo studio, comunque, non può limitarsi alle sole opere

\footnotetext{
" Cfr. B. ChiESA, "Shem Tob ibn Falaquera traduttore di al-Fārābī e di Averroè», Sefarad 49 (1989) 21-35.

7 J. Brugman - H. J. Drossaart Lulofs (edd.), Aristotle. Generation of Animals: the Arabic Translation Commonly Ascribed to Yahyā ibn al-Bitrīq, Leiden 1971.
} 
autentiche del filosofo: non minore deve infatti essere la considerazione riservata sia agli scritti "pseudoepigrafi" (che il Medioevo conobbe e ampiamente sfruttò), sia a quelli che, benché notoriamente non-aristotelici, vennero considerati come indispensabile completamento dell' «enciclopedia» scientifica dello Stagirita. A quest'ultimo gruppo di opere va ascritto anche il De plantis del peripatetico Nicola di Damasco (floruit 20 a. C.). La fortuna di quest'opera, che faceva probabilmente parte del Compendio della filosofia di Aristotele dello stesso autore, è dovuta infatti alla perdita dell'analoga opera botanica redatta da Aristotele, della quale lo scritto di Nicola, secondo le liste arabe delle opere aristoteliche, altro non sarebbe che un tafsirr (da intendersi più come "compendio» che come "commento»). L'opera, in realtà, è un trattatello di botanica, nel quale è

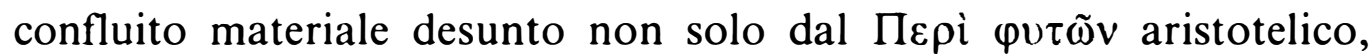
ma anche dalla Historia plantarum e dal De causis plantarum di Teofrasto (il cui testo greco ci è stato conservato).

A sua volta, il De plantis ebbe una storia travagliata. Andato perduto il testo originale, restano di esso solo alcuni frammenti della versione siriaca e la traduzione araba (completa), dalla quale vennero successivamente tratte le due versioni ebraiche (una delle quali compendiosa) e quella latina; quest'ultima subi poi la sorte curiosa di una "retroversione" greca, che venne addirittura inserita nel "canone» dell'opera omnia di Aristotele (pp. 814a-830b dell'ed. I. Bekker).

Quanto mai opportuna è dunque l'edizione che di queste traduzioni hanno recentemente curato Drossaart Lulofs e E. Poortman ${ }^{8}$. Il primo dei due studiosi è, tra l'altro, ben noto, in campo orientalistico, per i suoi approfonditi e fruttuosi studi sulla tradizione araba e siriaca delle opere zoologiche di Aristotele, che lo hanno condotto alla preparazione di un'edizione critica del De generatione animalium greco (Oxford 1965) sulla base di tutta la documentazioane orientale rintracciabile (nella scia delle edizioni di L. Minio Paluello per i primi libri dell'Organon). A sua cura vengono qui pubblicate le traduzioni siriaca, araba e ebraica del De plantis ${ }^{9}$

\footnotetext{
${ }^{8}$ Tale edizione era stata preannunciata da $\mathrm{H}$. DAIBER nella sua rassegna critica "Semitische Sprachen als Kulturvermittler zwischen Antike und Mittelalter. Stand und Aufgaben der Forschung», ZDMG 136 (1986) 292-313: 303 n. 58; cfr. 307 n. 80.

${ }^{9}$ Lo stesso H. J. DrossaART Lulofs aveva già sottolineato l'importanza delle

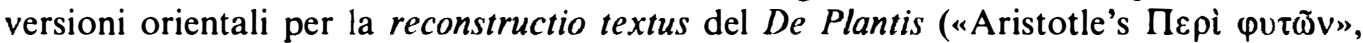
JHS 77 [1957] 75-80). Sulla fortuna degli scritti botanici della tarda antichità negli ambienti siri e arabi, v. anche J. F. HABBI, "Testi geoponici classici in siriaco e in
} 
mentre l'edizione del testo latino e di quello greco sono di competenza di Poortman.

Questa monumentale opera mira non solo a presentare in edizione critica tutti i frammenti di una qualche entità delle versioni medievali del De plantis, ma anche a definine i rapporti, sia per garantire una più sicura restitutio textus di ciascuno di essi, sia per chiarire nei dettagli la storia e la fortuna dell'opera (per facilitare il confronto, gli editori hanno mantenuto la stessa divisione in paragrafi per tutte la versioni). Il libro si articola pertanto in cinque grandi parti, dedicate rispettivamente a:

1) "The Fragments of the Syriac Translation [ $\Sigma]$ " (pp. 17-113);

2) "The Arabic Translation" (pp. 115-345);

3) "Translations and Commentaries in Hebrew" (pp. 347-463);

4) "The Latin Translation" (pp. 465-561);

5) "The (Anonimous) Greek Translation» (pp. 563-652).

La versione araba, la traduzione ebraica "compendiosa" e tutti i testi editi che non dipendano direttamente dalla prima sono utilmente accompagnati dalla traduzione inglese, posta a fronte. Il tutto è preceduto da una introduzione (pp. 1-16), che delinea rapidamente le vicende dello studio del De plantis in età moderna, ed è seguito da ricchi e utilissimi indici dei termini siriaci, arabi, latini e ebraici (pp. 666-729) '".

Già nella struttura, l'opera di Drossaart Lulofs e di Poortman, che, condotta con encomiabile rigore filologico e storico, sa ben chiarire le principali vecende della fortuna di questo scritto, merita di essere presa a modello per le future edizioni dell'Aristoteles Semitico-Latinus.

Passeremo ora in rapida rassegna i contenuti di ciascuna sezione dell'opera, per soffermarci poi con più attenzione sulla terza, dedicata alla tradizione ebraica.

\section{La versione siriaca}

Il testo completo di questa traduzione è -apparentementeandato perduto. In effetti, il solo ms che conservi una versione

arabo", in G. FIACCADORI (ed.), Autori classici in lingue del Vicino e Medio Oriente, Roma 1990, 77-92.

10 L'indice dei termini ebraici, per esigenze tecniche, è stato ridotto ai soli nomi proprî e al lessico botanico. 
siriaca del Compendio della filosofia di Aristotele (Cambridge, Univ. Libr., Gg. 2.14) non contiene altro che sparsi frammenti di questo scritto ", al punto che del De plantis restano solo poche frasi: esse sono tutte contenute nel f. 383 di dettro codice, che viene riprodotto in edizione diplomatica alle pp. 50-53 ${ }^{12}$. Ben più consistenti sono invece $\mathrm{i}$ frammenti che di questa versione si leggono nelle opere dell'ultimo esponente della letteratura siro-giacobita, Gregorio BarHebraeus (1226-1286). Drossaart Lulofs ha dunque provveduto a recuperare i brani del De plantis contenuti in due opere di questo scrittore: Menōrat Qūdše (Il Candelabro del Santuario), una grandiosa summa theologica composta secondo il più classico metodo scolastico, nella cui «base» dedicata all' «esamerone» si trova un capitolo sulla botanica costituito appunto da excerpta del nostro testo (edizione alle pp. 56-63) ${ }^{13}$; e Hewāt Hekmtā (La Crema della Sapienza), una altrettanto grandiosa enciclopedia aristotelica, pressoché totalmente inedita, della quale viene qui pubblicato l'intero libro De plantis, sulla base di sette mss (pp. 68-113). La sostanziale fedeltà di questi estratti al testo di Nicola è garantita proprio dal carattere compilatorio degli scritti di Bar Hebraeus; purtroppo, essi si limitano al primo dei due libri di cui si compone l'opera.

\section{La versione araba}

La versione araba del De plantis, alla quale si rifanno tutte le altre versioni note (eccettuata naturalmente quella siriaca, che ne è la diretta antenata), è generalmente attribuita a Isḥāq ibn Hunayn († 911), e sarebbe stata successivamente corretta da Tābit ibn Qūrrah († 901). Il suo testo venne per la prima volta scoperto da $\mathbf{M}$.

${ }^{11}$ Cfr. l'edizione dei primi cinque libri del Compendio fatta dallo stesso H. J. DrossaART Lulofs: Nicolaus Damascenus on the Philosophy of Aristotle, Leiden 1961 $[=1969]$.

12 L'editore avanza il dubbio che il De plantis non abbia in realtà fatto parte del Compendio, come invece aveva in un primo tempo sostenuto (v. H. J. DrossaART Lulofs, On the Philosophy, p. 40), o perlomeno che il testo si sia frantumato assai presto nelle sue diverse parti.

13 Il testo di questo capitolo era già stato edito una prima volta (in modo del tutto insoddisfacente) da $\mathrm{R}$. GoTTHEIL in un libretto scritto per il settantesimo genetliaco del celebre bibliografo M. Steinschneider (A List of Plants and their Properties from the Měnāreth Kudš̄ē of Gregorius Bar 'Ebhrāyā, Berlin 1886); il testo di tutta la "base» venne più tardi edito da J. BAKoš nel 1933 (PO 24.3), secondo tre dei cinque mss dell'opera consultabili nelle biblioteche occidentali. 
Bouyges nel 1922 nel ms di Istanbul, Yeni Cami $1179^{14}$, e, sulla base di questo solo testimone (confrontato con la versione latina di Alfredo di Sareshel), venne edito due volte, da A. J. Arberry nel 1933-1934 ${ }^{\text {is }}$ e da 'A. Badawi nel $1954{ }^{16}$. Nel frattempo, tuttavia, le testimonianze di quest'opera si sono arricchite di altri quattro mss (scoperti a Teheran e a Taškent): grazie a tre di essi (si è escluso solo il ms di Teheran, Malek 4655, del quale l'editore non ha potuto disporre) Drossaart Lulofs è stato in grado di approntare una nuova edizione critica (pp. 126-215). Per questa edizione, inoltre, sono state prese in considerazione le versione ebraiche e latina, nonché le lezioni riscontrabili negli estratti del De plantis impiegati dal medico e filosofo nestoriano Abū-l-Farağ Ibn al-Ṭayyib ( $† 1043)$ nel suo trattato di botanica. In tale trattato, conservato nell'unicum dell'Escoriale, Derenbourg 888, Ibn al-Tayyib rielabora materiali di varia origine: i passi dell'opera di Nicola sono comunque tutti ripresi nei capp. 24-29 (pubblicati alle pp. 219-231). Anch'egli, tuttavia, come il suo contemporaneo Avicenna (che impiega il De plantis - pur senza mai citarlo alla lettera- per il libro VI della sezione dello $\check{S}$ if $\bar{a}^{\prime}$ dedicata alle scienze naturali) ${ }^{17}$, mostra di non conoscere il secondo libro dell'opera.

\section{La versione latina}

La versione latina -opera di Alfredo di Sareshel (?11601225?) ${ }^{18}$ - ebbe la sua editio princeps a Venezia nel 1482, ma rimase poi per lungo tempo ignorata dagli studiosi, che vi preferirono la traduzione greca. Pubblicata infine da E. Meyer nel $1841^{19}$, e da U. C. Bussemaker nel 1857 (nell'ed. F. Didot delle opere di Aristotele, Parigi 1857, vol. IV), essa non era sino ad ora mai stata edita

\footnotetext{
14 "Sur le De plantis d'Aristote-Nicolas à propos d'un manuscrit arabe de Constantinople" MUSJ 9 (1923) 72-93.

15 "An Early Translation from the Greek", Bulletin of the Faculty of Arts of the Cairo University I (1933) 48-76, 219-257; II (1934) 71-83, 94-105.

${ }^{16}$ Aristatālis fi al-nafs, Cairo 1954, 241-281.

17 Edito da I. MADKūr, Cairo 1965 [= Qom 1404 eg.].

${ }^{18}$ I dati biografici e bibliografici relativi a quest'autore, estremamente scarsi e incerti, sono attentamente studiati da E. L. J. POORTMAN alle pp. 465-474.

${ }^{19}$ E. Meyer, Nicolai Damasceni de plantis libri duo Aristoteli vulgo adscripti..., Lipsiae 1841.
} 
criticamente. L'edizione di Poortman (pp. 515-561), dal canto suo, è fondata su sei boni codices, scelti dopo una accurata recensio dei circa $160 \mathrm{mss}$ di quest'opera. Tale disamina della tradizione giunge peraltro a interessanti conclusioni: in sostanza, della traduzione esistono due versioni, l'una corrispondente alla redazione preliminare - meno corretta stilisticamente, ma più aderente al dettato del testo arabo-, l'altra ad una successiva revisione, ascrivibile al traduttore stesso. D'altra parte, la presunta nova translatio dell'opera, rappresentata da due soli testimoni (il ms di Lipsia, Bibl. Univ., 1395, e quello di Parigi, B. N., 478), risulta essere nient'altro che un'ulteriore revisione della precedente.

La traduzione è accompagnata da un commento, redatto dallo stesso Alfredo, che Poortman non riproduce, rimandando all'edizione pubblicata recentemente da R. J. Long ${ }^{20}$.

\section{La versione greca}

Dalla traduzione alfrediana, intorno al 1300 , un anonimo erudito bizantino (da identificarsi con Massimo Planude, o forse, come suggerisce Poortman, col suo contemporaneo Manuele Holobolos) trasse una versione greca, che venne pubblicata in Occidente per la prima volta nel 1539, e poi più volte riedita nel Corpus Aristotelicum - per quanto il suo carattere spurio fosse stato denunciato già da Giulio Cesare Scaligero. In effetti, i molti passaggi del testo di Nicola attraverso lingue assai diverse per lessico e struttura, e le corruzioni generate dall'occasionale imperizia dei traduttori, rendono questa versione, ultima di una lunga catena, pressoché inutilizzabile: l'edizione che ne fa Poortman (pp. 589-624) ${ }^{21}$ ha pertanto un mero valore storico e documentario, quale testimonianza del rinato interesse per le opere dell'antichità nel Basso Medioevo greco.

\section{Le versioni ebraiche}

La tradizione ebraica del De plantis è particolarmente interessante, specialmente per la luce che getta su taluni aspetti della fortuna dell'opera in ambiente arabo, che resterebbero altrimenti in ombra.

${ }^{20}$ R. L. LONG "Alfred of Sareshel's Commentary to the Pseudo-Aristotelian De plantis: a Critical Edition", Medieval Studies 47 (1985) 125-167.

${ }^{21}$ Gli scoli che accompagnavano questa versione sono editi alle pp. 627-648. 
Una versione ebraica completa dell'opera venne redatta nel 1314 da Qalonymos ben Qalonymos ben Me'ir di Arles ${ }^{22}$. Per la sua edizione (pp. 407-435), Drossaart Lulofs si è basato su nove mss (di cui quattro frammentari):

$\mathrm{O}=$ Oxford, Bodl. Libr., Oppenheimer Add. 4.010 (spagnolo, sec. $\mathrm{XV}$;

$\mathrm{Z}=$ Paris, B. N., hébr. 1341 (italiano, 1555);

R = Parma, Bibl. Pal., parmense 2272 [= De Rossi 216] (spagnolo, inizio sec. XIV);

S = Parma, Bibl. Pal., parmense 2093 [= De Rossi 776] (italiano, fine sec. XIV);

$\mathrm{Y}=$ New York, J. T. S., n. 2444 (spagnolo, sec. XV);

$\mathrm{P}=$ Paris, B. N., hébr. 1005 (bizantino, sec. XV);

$\mathrm{K}=$ Budapest, Coll. Kaufmann 285 (bizantino o turco, fine sec. $\mathrm{XV}$;

$\mathrm{V}=$ Roma, Bibl. Ap. Vat., ebr. 290 (bizantino, sec. XV);

$\mathrm{A}=$ Oxford, Bodl. Libr., Hunt. 576 (spagnolo, inizio sec. XV).

Eccettuato l'ultimo ms, l'editore ha provveduto a classificare questi testimoni suddividendoli entro tre famiglie: $\alpha$ (abbracciante $\mathrm{O}, \mathrm{Z}$ e $\mathrm{R}$ ), che risulterebbe la più vicina all'archetipo - non presentando gravi errori testuali-; $\beta$ (comprendente $\mathrm{P}, \mathrm{K} \mathrm{e} \mathrm{V}$, e risalente a un sub-archetipo bizantino o turco, sottoposto a una non meglio precisata "revisione»); e $\gamma(=\mathrm{S}$ e $\mathrm{Y})$, che ha subito successive contaminazioni con le altre due famiglie; il $\mathrm{ms} \mathrm{Y}$, in particolare, incorpora nel testo numerose glosse.

\subsection{Un nuovo testimone della versione di Qalonymos}

Questa è la recensio completa dell'opera (discussa alle pp. 355361), secondo le informazioni che Drossaart Lulofs dichiara di aver ricevuto dall' «Institute of Microfilmed Hebrew Manuscripts» dell'Università di Gerusalemme. Come si vede, la documentazione è stata esaminata scrupolosamente, con una rigorosa applicazione dei metodi della critica testuale; le osservazioni che seguono, nelle quali si segnalano alcune integrazioni alle testimonianze raccolte dall'editore,

${ }^{22}$ Per questa traduzione, si vedano le indicazioni di M. STEInschneidir, Die hebraeischen Übersetzungen des Mittelalters, Berlin 1893 [Graz 1956] $[=H U ̈ b], \S 66$, 142-143, che per primo ne segnalò l'esistenza; per una bibliografia più recente sulla figura e l'opera di Qalonymos, cfr. G. TAMANI, «Il Corpus Aristotelicum nella tradizione ebraica", in G. FIACCADORI, op. cit., 33-46: 44-45. 
potranno essere utili per ancor meglio precisare l'importanza della tradizione ebraica del De plantis.

Per quanto riguarda, innanzitutto, la versione di Qalonymos, va rilevata l'esistenza di un altro testimone, il ms di New York, Jewish Theological Seminary, n. 8604 [d'ora in poi $=\mathrm{N}$ ], che pure si trova registrato nel catalogo dei microfilm dei mss filosofici conservati presso questa istituzione (A Reel Guide to Philosophy Manuscripts from the library of the Jewish Theological Seminary of America, Ann Arbor 1974, 23).

Secondo la sommaria descrizione offerta da questo catalogo, detto ms risale al sec. XIV, e venne scritto da mano italiana. Dunque, si tratta di uno dei più antichi testimoni dell'opera, insieme ai due mss di Parma (R e $\mathrm{S}$ ). Ecco quanto si può desumere dall'esame dei suoi contenuti:

Il ms contiene $115 \mathrm{ff}$, di 22 ll. ciascuno. Dal f. $1 \mathrm{v}$ al f. $98 \mathrm{r}$ si legge il testo (incompleto) dei Compendi di Averroè del De partibus [= P. A.] e De generatione animalium $[=\mathrm{G}$. A.], nella traduzione ebraica di Yacaqob ben Makir Ibn Tibbon ${ }^{23}$, secondo la seguente successione:

- 1r-20r: Compendio di P. A., libro 4 (= 14 secondo la numerazione complessiva delle opere zoologiche di Aristotele) [mutilo dell'inizio];

- 20r-40v: Compendio di G. A., libro $1(=15)$;

- 40v-61v: Compendio di G. A., libro 2 (=16);

- 61v-75v: Compendio di G. A., libro 3 (=17);

- 75v-89v: Compendio di G. A., libro 4 (=18);

- 89r-98v: Compendio di G. A., libro 5 (=19);

Dal f. 99r alla fine $(115 \mathrm{v})$, si trova invece il testo completo della versione del De plantis, di Qalonymos, sotto il titolo di : Ne indichiamo gli estremi:

Incipit del primo libro (99r1 ss.): אמר ארסטו החיות נמצא בבעלי חיים

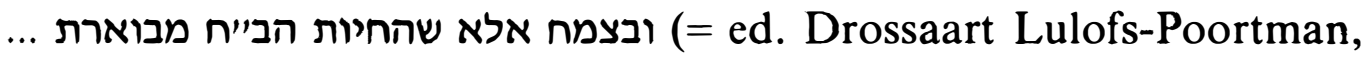
$407,1,1)$;

Incipit del secondo libro (107r7 ss.): דע שהצמח יש לו שלש כחות כח מסוג הארץ (= ed. Drossaart Lulofs-Poortman, 422, 135,1);

Colophon (115v1-5): נשלם המאמר השני מספר הצמחים ובהשלמו נשלם

${ }^{23}$ Per cui v. M. Steinschneider, HƯb. $\$$ 67, 144-145; A. Freimann, Union Catalogue, II, n. 1300; G. TAMANI, op. cit., 44. 
הספר והתחלה לאל והעתקתיו אני קלונימוס ברי קלונימוס ברי מאיר ...ed. Drossaart Lulofs-Poortman, 436).

$\mathrm{N}$ concorda generalmente con le lezioni della famiglia $\alpha$, alla quale indubbiamente appartiene, e, all'interno di quest'ultima, si accorda con le innovazioni proprie del sottogruppo $\mathrm{O}-\mathrm{Z}$. In particolare, esso presenta diverse omissioni comuni con $O, Z, R$ e $S$ (quest'ultimo, benché appartenga a $\gamma$, è stato contaminato con $\alpha$ ) ${ }^{24}$, e condivide con $\mathrm{i}$ primi due diverse lezioni alternative riportate a margine ${ }^{25}$ : è il caso, p. e., di הנפש in margine contro הספק (lez. corretta) nel testo (6,2), di שנוי (secunda manu in N e O) contro שוה (37,2), oppure di התאה in alternativa a $(91,3)$; ad O e Z lo accomunano anche talune aggiunte (p. e. quella di ויתחלק מהם: $48,4)$, che hanno il valore di errori congiuntivo-separativi rispetto al resto della tradizione.

\subsection{La traduzione di Falaquera: nuovi frammenti}

Ma la versione di Qalonymos non è l'unica — né la più importante- testimonianza della fortuna del De plantis in ambiente ebraico. Ad essa si aggiunge la più antica traduzione che Shem Tob ibn Falaquera riportò nella sua enciclopedia filosofica, Sefer $D e^{c} \hat{o} \underline{t} h a-$ Filosofim (Il libro delle dottrine dei filosofi), scritta verso il 1270. Per il vero, tale versione non venne tratta direttamente dal testo completo dell'opera, bensi da un compendio, che Falaquera attribuisce a non meglio identificati "Alessandrini" (אסכנדריים: v. p. 349). Si tratterebbe dunque di una Summa Alexandrinorum, analoga a quella redatta - probabilmente in greco, e poi tradotta in siriaco e in arabo- per l'Etica Nicomachea: Summa, quest'ultima, che Falaquera ben conosceva ${ }^{26}$. Tuttavia, l'esame del testo rivela che il compendio «alessandrino" del De plantis altro non è che un'epitome della versione araba: Drossaart Lulofs ipotizza dunque -e forse a ragione- che

${ }^{24}$ V. p. e. l'omissione di שעם (13,1), שלמה (21,5), di שתמם (52,2), ecc. Con $\mathrm{O}, \mathrm{Z}$ e $\mathrm{R}, \mathrm{N}$ ha in comune anche la caduta del colophon del l. I (v. p. 421 , apparato).

${ }^{25}$ È certo, peraltro, che $\mathrm{N}$ non coincide né con $\mathrm{O}$ né con $\mathrm{Z}$ in lectiones singulares; inoltre, nessuno di questi due mss risulta codex descriptus di $\mathrm{N}$.

${ }^{26}$ Come è stato recentemente dimostrato da B. CHIESA, "Una fonte sconosciuta dell'etica di Shem Tob ibn Falaquera: la Summa Alexandrinorum", in A. VIviAn (ed.), Biblische und judaistische Studien. Fs. für Paolo Sacchi, Frankfurt a. M. - Bern - New York - Paris 1990, 583-612. 
esso sia opera dello stesso (presunto) traduttore arabo della Summa Alexandrinorum dell'Etica, Abū 'A $\overline{1}$ 'Īsā ibn Zurcah (943-1008), e che, unito nello stesso volume di quest'ultima opera, sia stato erroneamente attribuito agli Alessandrini.

I frammenti della versione ebraica del "compendio" si trovano in massima parte nel capitolo primo del libro IV del Sefer Decôt , che l'editore pubblica alle pp. 388-405, sulla base dei due soli ms noti dell'opera (Parma, Bibl. Pal., Parmense $3156[=u]$ e Leiden, Univ. Libr., Or. $4758[=w])^{27}$. Tuttavia, è sfuggita all'attenzione di Drossaart Lulofs la presenza di altri due (seppur brevi) frammenti del testo nel 1. II, parte terza, capp. 5 e 9 dello stesso Sefer $D e^{c} \hat{o} \underline{t}$, אמר המחבר/המעתיק: ואמר/ממצא שאמר ארסטו בספר הצמח שscil. Falaquera]: afferma Aristotele nel libro De plantis ...". Per completezza, li pubblichamo qui sotto, indicando le eventuali varianti rispetto al testo arabo edito (la divisione del paragrafi riproduce quella dell'ed.):

$u$ 105a $18-24=w 172 \mathrm{va3}-12$ :

151 והצמח לא יקרה לו הרעש, מפני שהאויר בספוגות חלקיו. והראייה

על זה כי הרעש לא יהיה בחות לאות.

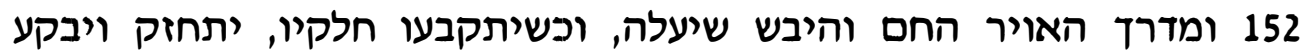

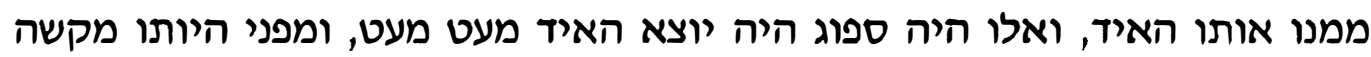

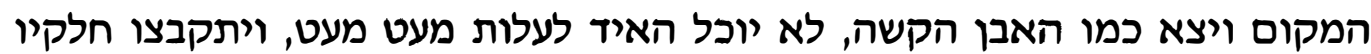

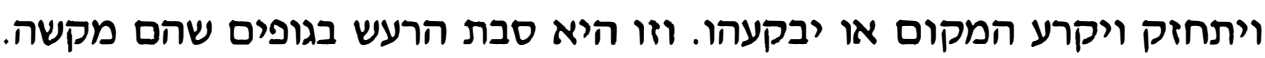

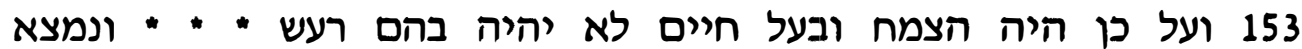

הרעש בחרס ובזכוכית.

Apparato:

Ebraico: 153 n' n'

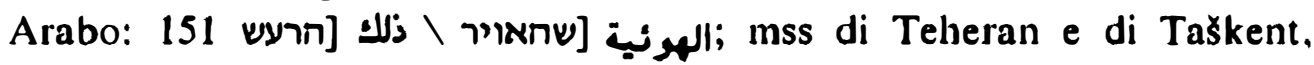

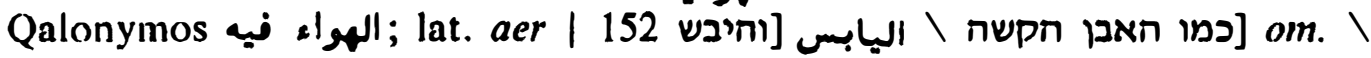
الحيران والنبات [הצמח اבעל חיים 153 |

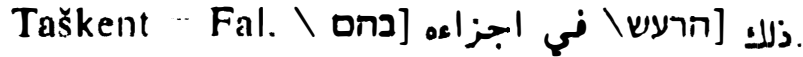

${ }^{27}$ Entrambi i mss sono in scrittura spagnola semi-corsiva, e risalgono rispettivamente al 1416 (non a "14th-15th cent.", come genericamente indicato da H. J. DrossaArt Lulofs a p. 353: si legga il colophon di detto ms, f. 293a) e al sec. XV(XVI). 
Traduzione:

"La pianta non subisce il terremoto, perché l'aria si trova nelle sue parti spugnose. Prova ne sia il fatto che il terremoto non avviene nella sabbia. Ed è natura dell'aria calda e secca di salire, e quendo le sue parti si riuniscono, essa si rafforza, spezza il luogo (dove si trova) e ne esce il vapore. Se (il luogo) è spugnoso, il vapore esce a poco a poco; ma quando è duro, come la pietra dura, il vapore non è in grado di salire a poco a poco: le sue parti si raccolgono, (esso) acquista forza e perfora il luogo o lo spezza. Questa è la causa del terremoto nei corpi duri. E per questo la pianta e l'animale non subiscono il terremoto, ma il terremoto si riscontra nella terracotta e nel vetro".

u 99b24-100a $7=w$ 169rb26-va8:

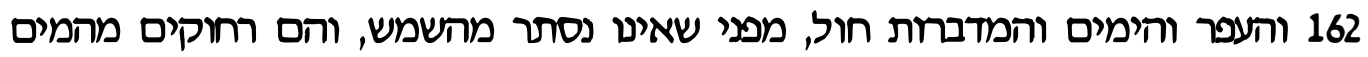

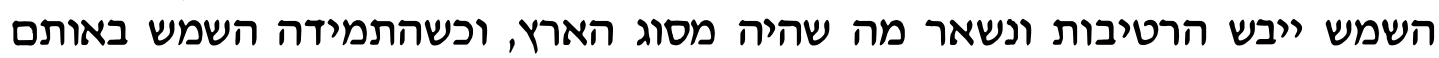
המתוקים, וחמימות המקומות ולא היו נסתרים ממנה, התפרקו חלקי וניותו הטיט ותיט והיה

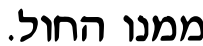

163 והראייה על זה כי כשנעמיק לחפור נמצא הטיט החורי. ותדע כי שורש * "

המקום מהמים המתוקים.

164 וכן מומר אמי כי מליחת מיחי מיח הים שרשם כולם המתוקות מהמים, ואמנם

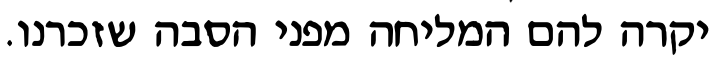

165 ואם אמר אומר כי הכולל מכל דבר הוא רובו ורוב המים מהים, ואם כן

הימים הם היסוד לכל המים.

[167-166] ונאמר כי המים המים המתוקים הם קלים מהמליחים ויותר טובים

מהם.

168 ועל כן היו המתוקים למעלה מהמימות כולם, והם רחוקים מטבע חמיע הארץ.

169 והמים הרחוקים מטבע הארץ הם הם המים הטבעיים מעים. ועל כן המים המים המתוקים

** * הם הטבעיים בהכרח.

Apparato:

Ebraico: המתיקות u [המתוקות 164 | חחולי יw [החורי 163 | שחוא

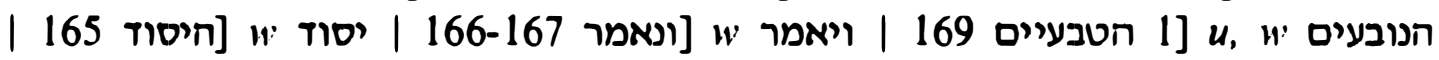

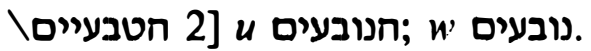

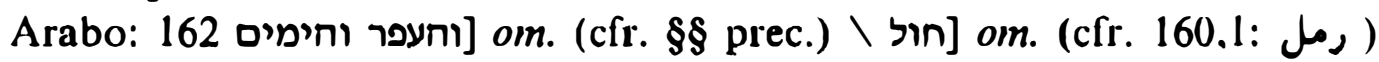

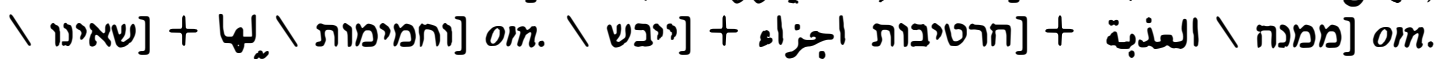

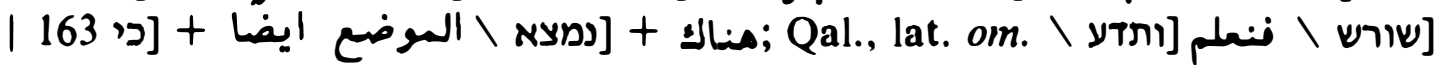

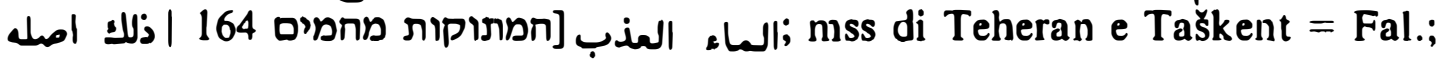

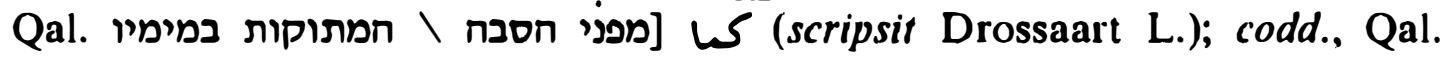

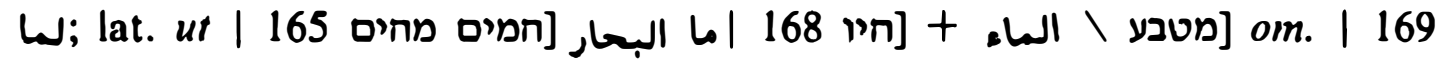

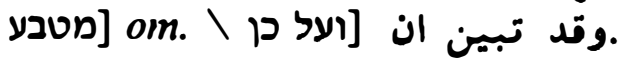


Traduzione:

"Il (terreno) arido, i mari e i deserti sono sabbia, perché non hanno protezione contro il sole, e sono lontani dall'acqua dolce; il calore del sole asciuga l'umidità, e resta (solo) ciò che è di genere terreo. E quando il sole persevera in quei luoghi che non hanno protezione contro di esso, le parti dell'argilla si separano e ne nasce la sabbia. Prova ne sia il fatto che, quando scaviamo in profondità, troviamo l'argilla pura. E sappi che alla base di (quel) luogo (c'è) l'acqua dolce. Si dice pure che l'acqua salata del mare (era) in origine tutta acqua dolce, e diventa salata per il motivo che abbiamo ricordato. E se qualcuno dice che la cosa (più) comune di ogni cosa è ciò che vi (si trova) in maggior quantità, e la maggior quantità d'acqua è nei mari, allora i mari sono l'origine di tutta l'acqua. E diciamo che l'acqua dolce è (più) leggera della salata, e più buona di quest'ultima. Per questo l'acqua dolce sta (più) in alto di tutta l'acqua, ed è (la più) lontana dalla natura della terra. L'acqua lontana dalla natura della terra è l'acqua naturale; perciò l'acqua dolce è, di necessità, naturales".

\subsection{Il commento di Averroè e un suo imitatore}

L'enciclopedia di Falaquera offre un'altra preziosa testimonianza. Come rilevato da Drossaart Lulofs, infatti, nel cap. terzo dello stesso libro IV (edito alle pp. 440-443), è contenuta una citazione di Averroè, che l'editore ritiene di poter identificare come un frammento del perduto Commento di Averroè al De plantis ${ }^{28}$. Tale commento (che risulta piuttosto un "compendio", simile per struttura al Compendio del De partibus $e$ De generatione animalium) era sinora noto - ma la sua esistenza era stata più volte messa in dubbio- solo da un sommario cenno in una lettera di Bernardo Navagero ${ }^{29}$, nonché da alcuni rimandi interni contenuti in altri commenti del filosofo cordovano. Ė poi proprio da Falaquera che Gershon ben Shelomoh trasse le citazioni «botaniche» di Averroè che si leggono nella terza "porta" della prima parte della sua enciclopedia di fisica e metafisica,

${ }^{28}$ L'effettiva correttezza di questa identificazione - che Drossaart Lulofs adotta troppo frettolosamente- non può essere qui discussa nei dettagli. Per la discussione in merito, rimandiamo il lettore a uno studio sulle fonti di tutto il libro IV delle $D e^{\varsigma} \hat{o} \underline{t}$, che ci proponiamo di pubblicare prossimamente.

${ }^{29}$ V. Aristotelis ... opera, ed. Giunta, Venetiis 1552, I, 20v: nuper enim Bernardus Naugerius ... librorum qui in ea urbe [Costantinopoli] apud Iudaeos \& Arabas medicos inveniuntur, hunc misit indicem. Tra i libri visti dal Navagero vengono menzionati anche i Magna Commentaria in libros duos de Plantis. 
שער השמים (La porta del cielo) ${ }^{30}$, le cui fonti vengono studiate da Drossaart Lulofs alle pp. 367-370.

Merita infine un cenno anche un ultimo brano "botanico" trasmesso da un anonimo autore ebraico, e contenuto nel già citato ms di Oxford (A). Si tratta di un frammento di una sorta di Commento grande al De plantis, che Steinschneider aveva ritenuto opera di Averroè ". Il fatto che i lemmi del testo commentato siano stati tratti direttamente della versione di Qalonymos fa tuttavia pensare che questo commento altro non sia che l'opera di un abile imitatore ebreo del filosofo spagnolo: conclusione cui Drossaart Lulofs giunge dopo un attento studio codicologico e filologico del ms (pp. 379386).

\section{LA FILOLOGIA EBRAICA MEDIEVALE: DESIDERATA}

Dai due casi sopra esaminati si possono trarre -crediamoistruttive conclusioni sugli obiettivi che la filologia ebraica medievale dovrà porsi nel campo (assai spinoso, e per questo trascurato) dell'edizione dei testi, e di quelli filosofico-scientifici in particolare.

Da un lato, è certo che l'impiego costante e rigoroso dei principi ecdotici originariamente elaborati per la filologia classica e romanza è ormai imprescindibile. Questo comporta naturalmente non solo un superficiale uso degli strumenti tipici della critica lachmanniana (recensio, stemma codicum, ecc.), ma anche che le conclusioni preliminari assunte in sede teorica trovino poi effettiva applicazione nel concreto approntamento dell'edizione (come non sempre è avvenuto -lo si è visto- nel caso dell''Iggeret ha-Wikkûah).

D'altro lato, tuttavia, la letteratura, ebraica del Medioevo - che $\grave{e}$, in non piccola parte, letteratura di traduzioni (dall'arabo o dal latino) - comporta problemi peculiari. Sta infatti emergendo l'importanza di tante sue opere per la storia e la critica testuale dei testi filosofici e scientifici arabi e greci (un caso perspicuo è appunto quello del De plantis): queste opere vengono pertanto vagliate e

30 V. M. STEINSCHNEIDER, HƯb. \& 3, 9-16.

${ }^{3}$ HUb. $\S 66,142-143$. Steinschneider aveva creduto che questo commento fosse contenuto (per intero) anche nei mss $\mathrm{R}, \mathrm{S}$ e $\mathrm{K}$ : in realtà, come dimostra Drossaart Lulofs, detti codici contengono solo la traduzione ebraica di Qalonymos (v. una più dettagliata discussione alle pp. 373-379). 
sfruttate per ricavarne varianti, tracce di redazioni e recensioni non altrimenti note, frammenti tratti da commenti perduti, ecc. Tale sfruttamento - che è pure utilissimo per gli storici della scienza e della filosofia- comporta però lo svantaggio di trascurare l'unità e l'organicità delle opere prese in considerazione (è questo il caso soprattutto di grandi compilazioni enciclopediche, come le stesse Dé $e^{\mathrm{t}}$ ha-Filosofim). Sarebbe invece auspicabile che questi testi, per quento vasti essi siano, fossero pubblicati nella loro interezza, in edizioni critiche che, seguendo l'esempio della pubblicazione di Drossaart Lulofs e Poortman ora presentata, segnalassero in apparato non solo le varianti interne della tradizione manoscritta (diretta e indiretta), ma anche quelle desumibili dai rapporti con le fonti dirette, e che fossero munite di dettagliati indici dei termini tecnici, consentendo cosi uno studio comparativo del lessico scientifico delle «lingue letterarie» del Medioevo orientale e occidentale, a tutto vantaggio di una migliore comprensione della lingua ebraica medievale ${ }^{32}$.

Un rigoroso lavoro di confronto con le fonti - che vale, a parer nostro, non solo per le traduzioni e le compilazioni, ma anche per gli scritti cosiddetti "originali»- non è motivato da mania filologistica o antiquaria, ma è spesso il solo che possa dar contezza al lettore dell'interesse di una letteratura, che raggiunge i suoi più alti risultati laddove riesce a realizzare un sapiente eclettismo tra diverse radici culturali: e l'opera di Falaquera è, sotto questo punto di vista, davvero esemplare.

${ }^{32}$ Edizioni di testi ebraici cosi concepite sono state già sperimentate -com’è noto-dalla "Medieval Academy of America", che ha pubblicato le versioni ebraiche di alcuni dei Commenti aristotelici di Averroè (se ne veda una rapida rassegna in Henoch 5 [1983] 409-412 [B. CHIESA]); altri problemi sono tuttavia suscitati dalla presenza di "redazioni plurime" - vere e proprie "varianti di traduttore»- in queste versioni, per cui cfr. le osservazioni di G. TAMANI, "Preliminari metodologici all'edizione di testi ebraici medievali", in G. FIACCADORI (ed.), op. cit., 207-214: 213 ss. 


\section{RESUMEN}

La reciente publicación de dos libros ofrece la ocasión de profundizar en algunos aspectos de la función de Shem Tob ben Yosef ibn Falaquera, filósofo sefardí del siglo XIII, como divulgador y traductor de las obras filosóficas y científicas árabes. Se trata de las ediciones (acompañadas de traducción inglesa) del Iggeret ha-Wikuah, a cargo de S. Harvey, y de la sección del Sefer Dé $\hat{t} \underline{t}$ ha-Filosofím dedicada al De plantis de Nicolás de Damasco, a cargo de H. J. Drossaart Lulofs. Se examinan los criterios editoriales y las bases adoptadas en cada una de las ediciones, y se hacen algunas sugerencias sobre correcciones y adiciones que podrían incorporarse.

\section{SUMMARY}

The recent publication of two books gives the opportunity of a study about some aspects of the work of Shem Tob ben Yosef ibn Falaquera, a Sephardi philosopher living in the 13th century, as translator of Arabic philosophy and science. They contain editions (accompanied by English translations) of two writings: the 'Iggeret ha-Wikuah, edited by S. Harvey, and the section of the Sefer Détôt ha-Filosofim devoted to Nicolaus Damascenus' De Plantis, edited by H. J. Drossaart Lulofs. The ecdotic criteria and the textual basis of the two editions are examined, and some corrections and integrations are suggested. 